\title{
BMJ Open Clinical communication in inflammatory bowel disease: a systematic literature review protocol
}

\author{
Neda Karimi (D) , ${ }^{1,2}$ Alison Rotha Moore, ${ }^{3}$ Annabelle Lukin, ${ }^{4}$ Ria Kanazaki (D) , ${ }^{1,5}$ \\ Astrid-Jane Williams, ${ }^{1,5}$ Susan Connor ${ }^{1,5}$
}

To cite: Karimi N,

Moore AR, Lukin A, et al.

Clinical communication in

inflammatory bowel disease:

a systematic literature

review protocol. BMJ Open

2020;10:e039503. doi:10.1136/

bmjopen-2020-039503

- Prepublication history and additional material for this paper is available online. To view these files, please visit the journal online (http://dx.doi.org/10. 1136/bmjopen-2020-039503)

Received 17 April 2020 Revised 31 August 2020 Accepted 26 September 2020

Check for updates

(c) Author(s) (or their employer(s)) 2020. Re-use permitted under CC BY-NC. No commercial re-use. See rights and permissions. Published by BMJ.

For numbered affiliations see end of article.

Correspondence to

Dr Neda Karimi;

Neda.Karimi@unsw.edu.au

\section{ABSTRACT}

Introduction Evidence regarding effective communication between clinicians and patients with inflammatory bowel disease (IBD) is limited. Studies that investigate clinical communication in IBD are much fewer in number than studies that investigate the perceptions of patients and clinicians about communication in clinical encounters. The current review aims to identify, organise and summarise systematically what is currently known about (1) the characteristics of interactions between clinicians who manage IBD and patients with IBD, and (2) how clinical discussion affects health outcomes in IBD.

Methods and analysis Scopus, PubMed, Embase, Communication Abstracts, Health \& Society, Linguistics and Language Behavior Abstracts and PsycINFO will be systematically searched for studies that investigate the characteristics of IBD clinical interactions during recorded consultations, from earliest available dates within each database to May 2020. A specifically developed quality assessment tool, grounded in linguistic theory, will be used to critically assess the evidence. In addition, a data extraction template will be developed and utilised to provide a description of the characteristics of IBD clinical communication as well as an estimation of its effect on health outcomes in a narrative synthesis.

Ethics and dissemination Ethical review and approval is not required for this systematic review as no primary data will be collected. The results will be published in peerreviewed journals and presented at academic conferences. PROSPERO registration number International Prospective Register of Systematic Reviews (PROSPER0) on 28 April 2020 (registration number: CRD42020169657).

\section{INTRODUCTION}

Inflammatory bowel disease (IBD) is a chronic inflammatory condition of the gastrointestinal tract mainly presenting in two forms: Crohn's disease (CD) and ulcerative colitis (UC). IBD is characterised by intermittent periods of active disease with symptoms including diarrhoea, rectal bleeding, urgency, incontinence, chronic abdominal pain, loss of appetite and weight loss, fatigue, joint pain and skin problems that undermine patients' quality of life and emotional wellbeing which can affect their personal, social

\section{Strengths and limitations of this study}

This systematic review will be the very first to identify, assess and summarise evidence resulting from investigations of recorded clinical interactions during IBD consultations.

- The review will consult a diverse range of databases-including databases with special focus on medicine, health, psychology, communication and linguistics - to identify eligible studies.

- The review will use a specifically developed quality assessment tool, grounded in linguistic theory, to critically assess the evidence.

- It is expected that the findings will not be integrated to produce cumulative evidence due to the anticipated diverse range of included studies in terms of context and theoretical underpinnings.

- Due to funding limitations, this systematic review will be restricted to publications in English language only and, thus, may not represent all the available evidence.

and professional life. The incidence of IBD is highest among those aged between 15 and 29 years, ${ }^{1}$ exacerbating the economic burden of the disease due to effects on the ability to work of the large young population of patients with IBD.

Due to the chronicity of IBD, patients require ongoing monitoring and long-term maintenance therapy to stay in remission and prevent recurrence of disease activity. Treatment of IBD has become more effective over time due to advances in medical and clinical research and the introduction of more effective drugs. At the same time, it has become more complicated because of the complex risk-benefit profile of the more effective treatments. As a result, discourses around the role of the patient as a key stakeholder in decision-making have found more recognition and prominence in IBD research. ${ }^{23}$ Since the main space in which clinicians and patients negotiate roles and make decisions is their clinical interaction during consultations, 
understanding the exchange of meaning between clinicians and patients in this space and its existing variations is crucial for understanding the bigger picture of howand how well-IBD is managed. Such an understanding can help identify ways in which IBD care can improve.

Effects of clinical communication on health outcomes include patient satisfaction, adherence, patient quality of life, disease management and self-management, as discussed by a number of studies in the IBD-specific literature and by many more studies concerned with other conditions. Ghosh and colleagues argued that in IBD, 'good communication between physician and patient is a cornerstone of effective disease management ${ }^{4}$ ( $p$ $\mathrm{S} 245$ ). The authors suggested that motivational communication may be valuable in IBD care, 'where the use of treatments with potentially undesirable side effects must be balanced against the risk of life-long high morbidity from the disease" (p S247). Motivational communication is a collaborative approach used to elicit the person's own intrinsic motivation and resources for change. ${ }^{5}$ A survey study by Mocciaro and colleagues showed that motivational communication in IBD consultations improved patient satisfaction, and potentially medication adherence and smoking cessation and helped physicians in dealing with patients 'moving from 'cure' to 'care". 6

Highlighting the link between clinical communication and patient quality of life and disease management, Mitchell and colleagues argued that discussing the impact of IBD on a patient's daily life during a consultation can produce a better 'picture of how patients are affected by their disease and how well their current treatment strategy is working for them ${ }^{, 7}$ (p2), and provides a context for considering new treatment options based on patients' expectations of treatment, ability to adapt and treatment objectives. Furthermore, Kennedy and colleagues pointed out the impact of effective communication on 'encouraging and supporting decisions and self-care actions which may enable patients to optimally manage their condition outside of health service settings ${ }^{8}$ (p567-8).

While there has been advocacy for research on communication in IBD, projects whose 'site of engagement/ intervention' is the 'clinician-patient interface ${ }^{9-}$ that is, projects that investigate interactions between patients and clinicians, rather than patients' perceptions of clinical communication-are less known. No systematic literature review has been conducted to identify and review such studies. In 2004, Husain and Triadafilopoulos pointed to 'a paucity of data concerning effective communication methods enabling physicians to develop stronger rapport with patients suffering from IBD ${ }^{10}$ (p444). Sixteen years later, we still do not know much about the status of IBD communication from research that uses real-life clinician-patient conversation data. The current review aims to ascertain the existing knowledge in this area to inform the field, identify the gaps and areas that require further investigations, and position this literature within current IBD care practice and research. The main objective is to identify, organise and summarise systematically what is currently known about (1) the characteristics of conversations between clinicians who manage IBD and patients with IBD, and (2) how clinical discussion affects health outcomes in IBD.

\section{METHODS}

The development of this study protocol was in accordance to the Preferred Reporting Items for Systematic Review and Meta-Analysis Protocols (PRISMA-P). ${ }^{11}{ }^{12}$ A copy of the completed PRISMA-P 2015 checklist is presented in online supplemental appendix 1. This study protocol is registered with the International Registration of Systematic reviews (PROSPERO, registration number: CRD42020169657).

\section{Eligibility criteria}

The review will include studies that investigate the characteristics of the interactions between clinicians who manage patients with IBD and/or their parent/guardian during a recorded consultation. These characteristics generally include, but are not limited to, the content of the consultation, patients' and clinicians' experience as represented in their language, the interpersonal meanings exchanged in the consultation, the different rhetorical steps that make up the consultation and the flow of information in the consultation. Studies based only on self-report of interaction for example, focus group studies, interviews, surveys or participatory observation with no audio/video recording will be excluded.

Published peer-reviewed studies in English that used quantitative or qualitative methods (including, but not limited to, discourse analysis, conversation analysis and content analysis) to analyse recorded real-life interactions between clinicians and patients with IBD (UC or $\mathrm{CD}$ ) during a consultation will be included in the review. Eligible studies will need to sample patients with IBD and clinicians who manage patients with IBD in primary and secondary healthcare (eg, general practitioners, IBD specialists, IBD nurses), complementary medicine (eg, acupuncturists, traditional Chinese medicine practitioner) or allied health (eg, dietitian). Studies with a focus on healthcare providers whose primary treatment includes the interaction itself (eg, psychotherapists) will be excluded. Studies in which these participant groups are present but IBD is not the focus of the study will also be excluded. Studies will be selected regardless of the type of intervention or exposure as the review will not be focused on a certain type of intervention or exposure. Only journal articles and book chapters published in English are eligible. Peer-reviewed published abstracts, letters to the editor, editorials and theses will be excluded. However, ineligible sources will be examined to locate corresponding journal articles. Articles published up to May 2020 will be included.

Information sources and search strategy

The review will search for records indexed in:

1. Scopus 
2. PubMed

3. Embase

4. Communication Abstracts

5. Health \& Society

6. Linguistics and Language Behavior Abstracts

7. PsycINFO

In addition, snowball sampling will be employed. Reference lists of eligible articles identified in the online database search as well as the excluded but relevant publications will be consulted. Subject matter experts (those known to the researchers as well as those identified in the database search and snowball sampling) will be contacted via email and consulted to identify any additional literature.

A relatively broad search strategy will be employed due to anticipating limited numbers of studies that explore real-life clinician-patient interactions in IBD and in order to maximise the reach. Table 1 lists the keywords that will be used to search these databases. Keywords referring to the condition or healthcare domain being studied (eg, IBD) will be used; in conjunction with terms describing the data type (eg, consultation and audio-record*). The search strategy will represent the intersection of these two sets of terms.

\section{Data management and selection process}

Study records obtained from the databases will be exported into Endnote where duplicates will be removed, and screening of titles and abstracts and then full-text records will be performed independently by three reviewers (NK, RK and AL). The reviewers will be overinclusive with their selections and will include all the studies that appear to meet the inclusion criteria as well as those whose eligibility for inclusion is uncertain. Reviewers will not be blinded to the study authors, institutions or journals of the records they screen.

Once the reviewers complete the screening of titles and abstracts, they will meet to compare their lists of selected studies and resolve any discrepancies prior to the full-text review. Any unresolved disagreement will be discussed with the whole review team and a collective decision will be made. Reasons for exclusion will also be recorded at this stage. Once agreement is reached, the full text of the selected studies will be uploaded in Endnote and studied independently by the reviewers for final inclusions. The same discrepancy resolving process will be repeated at this final stage of selection. Reviewers will meet on finishing the independent selection process to resolve any disagreements and will discuss matters with the whole review team if they cannot reach an agreement.

\section{Data collection and extraction processes}

Selected articles will be carefully studied by the whole team. A data extraction template will be developed based on the questions asked in the review and the information available in the selected studies, and in consultation with the existing health communication and linguistics literature including previous systematic literature reviews of this kind ${ }^{913-16}$ and Halliday's theoretical model of the architecture of language, known as systemic functional linguistics. ${ }^{17}$ The data extraction template will be accompanied by detailed instructions in Microsoft Excel (2007). It will be piloted by two reviewers on a sample of included papers to ensure the efficiency of the template and the accuracy and consistency of extractors before the final data extraction which will be performed by NK and checked by the review team.

The review will explore potential trends in this strand of research by comparing the timing of studies (year of research), the countries in which the studies were conducted and the type of consultation under scrutiny (eg, IBD nurse consultations, IBD specialist consultations, etc). Information will be extracted on research setting, participant characteristics including their role (eg, patient, parent, nurse, gastroenterologist, etc), sociodemographics and the status of patient participants (eg, pregnant, preconception, postsurgery, in transition to adult care, etc), as well as disease characteristics including type of IBD (UC, CD or IBD unclassified), disease activity, disease phenotype and extraintestinal manifestations. Stated aims, aims relevant to the review (eg, investigation of whether/how the clinicians talk about treatment options including their benefits and side effects, patient's quality of life or goals of care; description of clinician-patient relationship as construed in talk; etc), study design, health outcomes and measures and stated findings and conclusions will be described for each study. Information on the consultation data including the size of the dataset (corpus size), the actual number of consultation/episodes analysed in the study, the average length of consultations, whether consultations were audio recorded or video recorded and whether the consultations were one-off or in series will be charted. Furthermore, the method of linguistic data analysis and the investigated linguistic features will be described. A linguistic feature is broadly defined as any semantic, grammatical or lexical concept such as topic, question (type and quantity), length of consultation and so on.

Box 1 outlines the data items that will be included in the review. Additional items will potentially be added to this list based on the information available in the selected papers.

\section{Outcomes and prioritisation}

A description of the characteristics of conversations between clinicians who manage IBD and patients with IBD (and/or their parent/guardian) during a consultation is the main outcome of this review. These characteristics generally identify the content of the consultation, patients' and clinicians' experience as represented in the consultation, the interpersonal relationships between clinician and patient, the different steps involved and the flow of information in the consultation. Another main outcome is an estimation of the effect of IBD clinical discussion on health outcomes (biomedical and psychosocial). Secondary outcomes include a description of the 


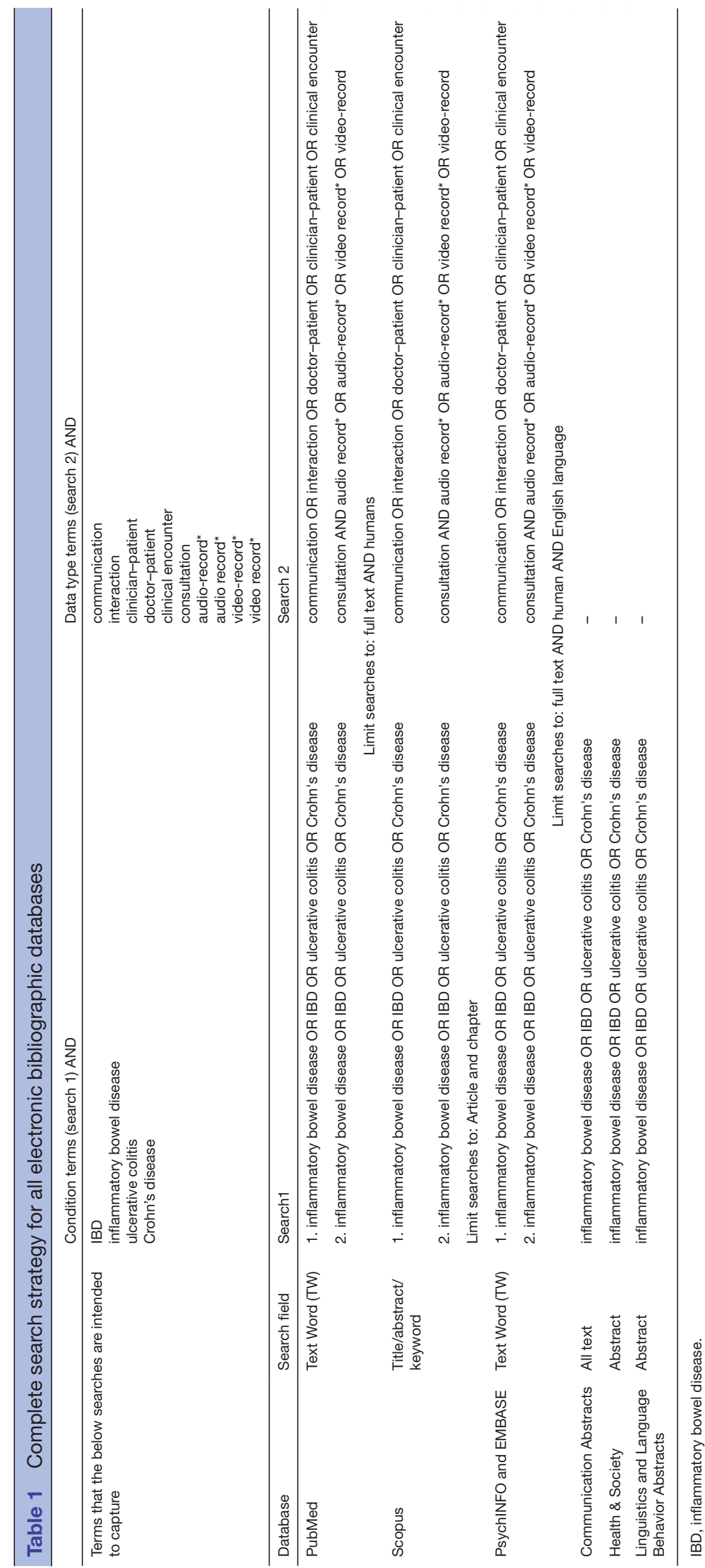

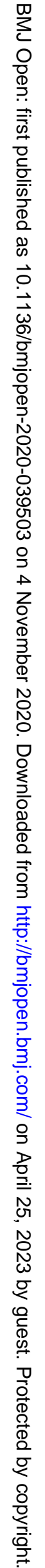


Box 1 Data items included in the data extraction template

Participant characteristics
Participants and numbers
Participant sociodemographics
Additional health status information
Disease characteristics
Type of IBD
Disease activity
Disease phenotype
Extraintestinal manifestation
Study characteristics
Year of research
Country of research
Research setting and type of consultation
Stated aims
Aims relevant to the review
Study design
Outcomes and measures
Stated findings
Stated conclusions
Consultation data and analysis characteristics
Corpus size and number of consultations/episodes analysed in the
study
Average length of consultations
One-off consultation or series
Data type (audio or video)
Method of linguistic data analysis (sociolinguistics, conversation
analysis, content analysis, etc)
Linguistic component/s analysed

characteristics of the existing consultation data available for scrutiny in the literature, and trends in IBD clinical communication research including mainstream analytic approaches.

\section{Risk of bias in individual studies}

Conventional guidelines for assessing the quality of studies for inclusion in a systematic literature review ${ }^{18}$ have limited application to discourse analytic research because this type of research is different from the mainstream qualitative and quantitative health research in terms of its objective and methodology. ${ }^{15}$ Rather than using a single set of criteria and ranking studies based on those criteria, following Parry and Land, two broad dimensions will be used to assess each study's value and contribution: (1) the type and amount of data, and (2) the credibility and reliability of the analysis. ${ }^{15}$ Credibility is defined as 'the confidence that can be placed in the truth of the research findings ${ }^{19}$ (p121). To assess the credibility of the studies, Matthiessen's account of the methodological approaches to the analysis of a situation type (eg, IBD consultation $)^{20}$ will be used as a guide. Matthiessen's methodological account ${ }^{20}$ is based on Halliday's systemic functional linguistics. ${ }^{21}$ Generally, language consists of four layers or strata (context, semantics, grammar and lexis and phonology) and four main functions (experiential, logical, interpersonal and textual). Function of language equals 'use': what is it that the language is being used for? The four main functions (or metafunctions) of language occur simultaneously in any utterance or text. ${ }^{17}$ The experiential function allows language users to use language to construe their experience; the interpersonal function allows language users to enact their roles and relationships with each other (eg, status, intimacy, contact, sharedness between interactants); the logical function concerns how language users create relations between different parts of their talk, and the textual function is what turns a collection of individual words into a coherent text. ${ }^{1722} \mathrm{~A}$ comprehensive description of a situation type is time consuming and labour intensive. Matthiessen suggests principled selection of data and data analysis tools to reduce the description bias and increase credibility. ${ }^{20}$ To assess the reliability of the studies, information regarding the presence or absence of a second coder and the use of a unit of analysis will be considered.

Included studies will also be evaluated in terms of the amount of evidence used to support their conclusions and whether the conclusions were biassed or evidence based. ${ }^{23}$ Further quality assessment dimensions may be added depending on the included studies. Missing information will not be sought from the authors, neither will unclear aspects of the studies be clarified with them. Rather, such limitations will be discussed under risk of bias.

\section{Synthesis}

The extracted data will be presented in overview tables for the purpose of summarisation and comparison and described in a narrative synthesis. The inclusion criteria in this review allow for including studies from a range of contexts such as IBD specialist consultations, nurse consultations, allied health consultations, and general practice consultations. It is, therefore, expected that the context of the included studies will vary. It is also expected that these studies will be within different research traditions, having different underpinning philosophical assumptions, given the diverse approaches to the analysis of talk in health research, in general. Considering the diversity of contexts and theoretical underpinnings, a narrative synthesis was chosen as the method of synthesising data.

The narrative synthesis will be based on the results of the data extraction and quality appraisal. Furthermore, following the recommendations of Cochrane Consumers and Communication Review Group, ${ }^{24}$ the narrative synthesis will also include investigation of the similarities and the differences between the studies based on the study design and information gathered from the data extraction and quality appraisal. Since this is not a metasynthesis, findings of the included studies will not be integrated, and the data will not be reinterpreted.

\section{Patient and public involvement}

There has been no contribution from patients or the public to the design of this systematic review protocol. 


\section{ETHICS AND DISSEMINATION}

No human subject participants will be involved. Therefore, ethical approval will not be required. Findings of this systematic review will be presented at national and international conferences and published in peer-reviewed journals (open access if possible). In the event of protocol amendments, the date of each amendment will be accompanied by a description of the change and the rationale.

\section{DISCUSSION}

Clinician-patient communication is shown to affect biological and functional health outcomes ${ }^{25-29}$ and can have economic consequences. ${ }^{30-32}$ In IBD, clinical communication is argued to affect patient satisfaction, treatment adherence, patient quality of life, disease management and self-management, as described in the Introduction section. This systematic review will be the first to review studies that examine clinical communication in IBD using recorded clinician-patient consultation data. It aims to investigate the characteristics of IBD clinical discussions and the effects of these discussions on health outcomes (biomedical and psychosocial). The current protocol outlines the steps and procedures involved in achieving this objective.

Collecting and reviewing evidence from studies that investigate recorded clinical communication in IBD for the first time, consulting a diverse range of databases to identify eligible studies, developing a broad search strategy to maximise inclusion and using a comprehensive theory of language for appraising the quality of the included studies are arguably among the strengths of this review. Nevertheless, there are limitations as well. Reviews of this kind inevitably include a diverse range of studies in terms of context and theoretical underpinnings and this review will not be an exception. The consequence of this diversity is that findings cannot be integrated to produce cumulative evidence. For this reason, a narrative synthesis approach will be taken where data will be summarised and compared but not statistically integrated. In addition, because of funding limitations, this review will be restricted to publications in English language only and, thus, may not represent all the available evidence. Nevertheless, the results of the review can provide clinicians with valuable information to improve the way they communicate with their patients during a consultation. It will also identify the gaps in the literature and the areas that require further investigation for future research.

\footnotetext{
Author affiliations

${ }^{1}$ Faculty of Medicine, South Western Sydney Clinical School, University of New South Wales, Sydney, New South Wales, Australia

${ }^{2}$ Gastroenterology Research Group, The Ingham Institute for Applied Medical Research, Liverpool, New South Wales, Australia

${ }^{3}$ Faculty of Law Humanities and the Arts, School of Humanities and Social Inquiry, University of Wollongong, Wollongong, New South Wales, Australia

${ }^{4}$ Faculty of Medicine, Health and Human Sciences, Department of Linguistics, Macquarie University, Sydney, New South Wales, Australia

${ }^{5}$ Department of Gastroenterology, Liverpool Hospital, Liverpool, New South Wales, Australia
}

Contributors NK, ARM and AL conceived the idea of this systematic review project. NK developed the protocol and prepared the first draft of this manuscript with feedback from ARM, AL, SC, RK and A-JW on the design of the protocol and the manuscript.

Funding The authors have not declared a specific grant for this research from any funding agency in the public, commercial or not-for-profit sectors.

Competing interests NK, ARM and AL have received grant support from Janssen. RK has received research and educational support from Pfizer, Abbvie, Takeda and Janssen. AW has received Honoraria from Takeda, Ferring, Janssen and Abbvie. SC has received Honoraria, speaker fees, educational support and/or research support from AbbVie, Celgene, Ferring, Gilead, Janssen, MSD, Novartis, Orphan/Aspen, Pfizer, Shire and Takeda.

Patient consent for publication Not required.

Provenance and peer review Not commissioned; externally peer reviewed.

Supplemental material This content has been supplied by the author(s). It has not been vetted by BMJ Publishing Group Limited (BMJ) and may not have been peer-reviewed. Any opinions or recommendations discussed are solely those of the author(s) and are not endorsed by BMJ. BMJ disclaims all liability and responsibility arising from any reliance placed on the content. Where the content includes any translated material, BMJ does not warrant the accuracy and reliability of the translations (including but not limited to local regulations, clinical guidelines, terminology, drug names and drug dosages), and is not responsible for any error and/or omissions arising from translation and adaptation or otherwise.

Open access This is an open access article distributed in accordance with the Creative Commons Attribution Non Commercial (CC BY-NC 4.0) license, which permits others to distribute, remix, adapt, build upon this work non-commercially, and license their derivative works on different terms, provided the original work is properly cited, appropriate credit is given, any changes made indicated, and the use is non-commercial. See: http://creativecommons.org/licenses/by-nc/4.0/.

\section{ORCID iDs}

Neda Karimi http://orcid.org/0000-0002-2841-637X

Ria Kanazaki http://orcid.org/0000-0002-8866-1078

\section{REFERENCES}

1 Johnston RD, Logan RFA. What is the peak age for onset of IBD? Inflamm Bowel Dis 2008;14 Suppl 2:S4-5.

2 Siegel CA, Lofland JH, Naim A, et al. Gastroenterologists' views of shared decision making for patients with inflammatory bowel disease. Dig Dis Sci 2015;60:2636-45.

3 Drescher H, Lissoos T, Hajisafari E, et al. Treat-to-Target approach in inflammatory bowel disease: the role of advanced practice providers. The Journal for Nurse Practitioners 2019;15:676-81.

4 Ghosh S, D'Haens G, Feagan BG, et al. What do changes in inflammatory bowel disease management mean for our patients? $J$ Crohns Colitis 2012;6 Suppl 2:S243-9.

5 Miller W, Rollnick S. Motivational Interviewing. In: Preparing people for change. Second ed. New York, UNITED STATES: Guilford Publications, 2002

6 Mocciaro F, Di Mitri R, Russo G, et al. Motivational interviewing in inflammatory bowel disease patients: a useful tool for outpatient counselling. Dig Liver Dis 2014;46:893-7.

7 Mitchell R, Kremer A, Westwood N, et al. Talking about life and IBD: a paradigm for improving patient-physician communication. $J$ Crohns Colitis 2009;3:1-3.

8 Kennedy A, Gask L, Rogers A. Training professionals to engage with and promote self-management. Health Educ Res 2005;20:567-78.

9 Moore AR. Language and medicine. In: Thompson G, Bowcher WL, Fontaine L, et al, eds. The Cambridge handbook of systemic functional linguistics. Cambridge: Cambridge University Press, 2019: 651-88.

10 Husain A, Triadafilopoulos G. Communicating with patients with inflammatory bowel disease. Inflamm Bowel Dis 2004;10:444-50.

11 Moher D, Shamseer L, Clarke M, et al. Preferred reporting items for systematic review and meta-analysis protocols (PRISMA-P) 2015 statement. Syst Rev 2015;4:1.

12 Shamseer L, Moher D, Clarke M, et al. Preferred reporting items for systematic review and meta-analysis protocols (PRISMA-P) 2015: elaboration and explanation. BMJ 2015;349:g7647.

13 Kindell J, Keady J, Sage K, et al. Everyday conversation in dementia: a review of the literature to inform research and practice. Int $J$ Lang Commun Disord 2017;52:392-406. 
14 Parry RH, Land V, Seymour J. Communicating face-to-face about sensitive future matters including end of life: a systematic review of evidence from fine-grained observational research Prospero 2011:CRD42011001626.

15 Parry $\mathrm{RH}$, Land V. Systematically reviewing and synthesizing evidence from conversation analytic and related discursive research to inform healthcare communication practice and policy: an illustrated guide. BMC Med Res Methodol 2013;13:69.

16 Stortenbeker I, Stommel W, van Dulmen S, et al. A review on linguistic and interactional aspects in consultations about medically unexplained symptoms. Prospero 2018:CRD42018095405.

17 Halliday MAK, Part AHalliday MAK, Hasan R, eds. Language, context, and text: aspects of language in a social-semiotic perspective. Oxford/Geelong: OUP/Deakin University Press, 1985/89.

18 Higgins JPT, Altman DG, Gøtzsche PC, et al. The Cochrane collaboration's tool for assessing risk of bias in randomised trials. BMJ 2011;343:d5928.

19 Korstjens I, Moser A. Series: practical guidance to qualitative research. Part 4: Trustworthiness and publishing. Eur J Gen Pract 2018;24:120-4.

20 Matthiessen C. Register in the round: Diversity in a unified theory of register analysis. In: Ghadessy $\mathrm{M}$, ed. Register analysis: theory and practice. London: Pinter, 1993: 221-92.

21 Halliday MAK, Matthiessen C. Halliday's introduction to functional grammar. London \& New York: Routledge, 2014

22 Halliday MAKWebster JJ, ed. On language and linguistics, volume 3 of the collected works of M.A.K. Halliday. London \& New York: Continiuum, 2003.

23 Aromataris E, Munn Z, eds. Joanna Briggs Institute Reviewer's Manual: The Joanna Briggs Institute, 2017.
24 Ryan R, Cochrane Consumers and Communication Review Group. Cochrane consumers and communication review group: data synthesis and analysis June 2013

25 Lee W, Noh Y, Kang H, et al. The mediatory role of medication adherence in improving patients' medication experience through patient-physician communication among older hypertensive patients. Patient Prefer Adherence 2017:11:1119-26.

26 Heisler M, Bouknight RR, Hayward RA, et al. The relative importance of physician communication, participatory decision making, and patient understanding in diabetes self-management. J Gen Intern Med 2002;17:243-52.

27 Street RL, Makoul G, Arora NK, et al. How does communication heal? pathways linking clinician-patient communication to health outcomes. Patient Educ Couns 2009;74:295-301.

28 Safran DG, Taira DA, Rogers WH, et al. Linking primary care performance to outcomes of care. J Fam Pract 1998;47:213-20.

29 Zachariae R, Pedersen CG, Jensen AB, et al. Association of perceived physician communication style with patient satisfaction, distress, cancer-related self-efficacy, and perceived control over the disease. Br J Cancer 2003;88:658-65.

30 Linedale EC, Chur-Hansen A, Mikocka-Walus A, et al. Uncertain Diagnostic Language Affects Further Studies, Endoscopies, and Repeat Consultations for Patients With Functional Gastrointestinal Disorders. Clin Gastroenterol Hepatol 2016;14:1735-41.

31 Hurtig RR, Alper RM, Berkowitz B. The cost of not addressing the communication barriers faced by hospitalized patients. Perspect ASHA Spec Interest Groups 2018;3:99-112.

32 Vermeir P, Vandijck D, Degroote S, et al. Communication in healthcare: a narrative review of the literature and practical recommendations. Int J Clin Pract 2015;69:1257-67. 